\title{
APRENDIZAJE INTERACTIVO EN ESTUDIANTES DE CONTABILIDAD DE LA CARRERA DE ADMINISTRACION DE EMPRESAS
}

\section{ANALYSIS OF INTERACTIVE LEARNING FOR ACCOUNTING STUDENTS IN CAREER OF BUSINESS ADMINISTRATION}

\author{
Rosa Benites Medina ${ }^{1}$, Marcelo Bastidas Jiménez ${ }^{2}$
}

\section{RESUMEN}

Las tecnologías de la información y comunicación fueron introducidas años atrás y paulatinamente han revolucionado los sistemas empresariales e incursionaron también en los entornos educativos, generando un nuevo modelo de enseñanza y aprendizaje, debido a que involucraron cambios en los procesos, objetivos, contenidos e infraestructura de las instituciones educativas que se van adecuando a las necesidades actuales de las empresas.

Con el presente artículo se pretende que los docentes de la asignatura de contabilidad de la carrera de Administración de Empresas, cuenten con acciones que contribuyan a elevar motivación en el estudio de la asignatura. Para ello fue necesario investigar y reflexionar en las causas de las elevadas tasas de reprobación de los estudiantes y de esa manera poder enfocar el problema con las metodologías adecuadas.

La metodología utilizada en el presente artículo fue de carácter mixto. El estudio cuantitativo, se llevó a cabo con la aplicación de una encuesta dirigida a los estudiantes que cursaron las asignaturas de Contabilidad 1 y 2 de la carrera de Administración de Empresas, durante el período 47. El estudio cualitativo, se llevó a cabo con la aplicación de entrevistas en profundidad dirigidas a los docentes de las asignaturas contables. En ambos casos los objetivos apuntaron a determinar la necesidad de tener tácticas que impliquen la utilización de nuevas tecnologías y del aprendizaje interactivo.

Los resultados confirman la necesidad de aplicar estrategias de aprendizaje interactivo, entre las cuales figuran las tutorías virtuales, la creación de videos tutoriales, la explotación de herramientas interactivas en los ambientes virtuales. Con esto se pretende, elevar la motivación de los estudiantes y disminuir las tasas de reprobación actualmente evidenciadas en la carrera.

Palabras claves: Aprendizaje interactivo, contabilidad, enseñanza.

Recepción: 10/01/2017. Aprobación: 11/10/2017.

\section{ABSTRACT}

The technologies of information and communication were introduced years ago and they have gradually revolutionized business systems and they have also reached educational environments, creating a new model of teaching and learning, because they involved changes at the processes, objectives, content and infrastructure of educational institutions that are adapting to the current needs of companies.

1 Universidad Politécnica Salesiana, Carrera de Administración de Empresas, Guayaquil, Ecuador. E-mail: rbenites@ups.edu.ec 2 Universidad Politécnica Salesiana, Carrera de Administración de Empresas, Guayaquil, Ecuador. E-mail: mbastidas@ups.edu.ec 
This article aims accounting teachers of the Business Administration career to have actions that allow their students to encourage the study of the subject. This required to research and to reflect in causes of the high failure rates of students and thus to approach the problem with appropriate methodologies.

The methodology used in this article was mixed. The quantitative study was carried out with the application of a survey directed to the students who attended the subjects of Accounting 1 and 2 of the career of Business Administration during the period 47. The qualitative study was carried out with the application of In-depth interviews directed to the teachers of accounting subjects. In both cases the objectives aimed to determine the need for tactics involving the use of new technologies and interactive learning.

The results confirm the need to apply interactive learning strategies, which include virtual tutorials, the creation of video tutorials and the use of interactive tools in virtual environments. This is intended to raise motivation of students and decrease failure rates currently evidenced in the career.

Keywords: Accounting, interactive learning, teaching.

\section{INTRODUCCIÓN}

El contexto de la educación en las asignaturas contables a nivel universitario en la actualidad es muy diverso, pese a que los organismos de control, dan pautas sobre los procesos metodológicos y evaluativos (Ministerio de Educación del Ecuador, 2016), se observa mucha heterogeneidad en la enseñanza propiamente dicha (Iglesias \& Iglesias, 2016). Es así como en algunos casos se sigue impartiendo la materia con ayuda de diapositivas y clases en pizarra, mientras en otros casos se utilizan laboratorios con Excel y en pocos casos se trabaja con sistemas contables.

La presente investigación se basa en la situación en que se encuentran los estudiantes que reciben la asignatura de contabilidad, donde se ha podido evidenciar la falta de motivación en el estudio con la estrategia de enseñanza actual, sumado al hecho de que la base de conocimientos con los que ingresan los estudiantes a esta carrera no es la adecuada para el normal desenvolvimiento de la cátedra.

La situación descrita desencadena una situación problemática en la carrera de administración de empresas de la Universidad Politécnica Salesiana Guayaquil, evidenciándose un alto índice de alumnos reprobados y repetidores en la asignatura de contabilidad, que se imparte a los estudiantes de primero y segundo semestre.

Ante este problema concreto se plantea la siguiente interrogante: ¿Qué tipo de acciones pueden desarrollarse por los docentes de la cátedra de contabilidad que, mediante las herramientas de aprendizaje interactivo, eleve la motivación del estudio de las asignaturas y disminuya los índices de reprobación y repitencia de las mismas?
En la actualidad la tecnología ha pasado a ser parte de la vida diaria de los estudiantes (Gallardo, 2012), por lo que sería necesario conocer de qué manera se podría utilizar las TICs, ligadas al aprendizaje interactivo, en beneficio del estudio de la materia de contabilidad en la carrera de Administración de Empresas.

El objetivo general de la presente investigación es realizar un análisis del aprendizaje interactivo en estudiantes de las asignaturas contables. Los objetivos específicos son por su parte: evaluar los niveles de aceptación de cambio de estrategia de la enseñanza contable impartida actualmente, analizar los niveles de reprobación en las asignaturas contables y Determinar algunas estrategias de aprendizaje interactivo que se podrían implementar bajo las circunstancias actuales.

El documento se encuentra estructurado de la siguiente manera, en primer lugar, se hace una aproximación al problema y una descripción general de lo que representan las tecnologías en la educación. Posterior a ello se hace una descripción de la metodología empleada, luego de lo cual se procede a presentar los resultados tanto de las encuestas, como de las entrevistas, sumado a una presentación de datos secundarios, que incluye estadísticas de los tres últimos periodos para establecer la cantidad de estudiantes reprobados y el análisis de pruebas de diagnóstico, para comprobación de las deficiencias académicas de los estudiantes. Finalmente, en la discusión se presentan algunas alternativas de herramientas y recursos que se utilizarían en la enseñanza contable. 


\subsection{El problema}

Actualmente la enseñanza se lo realiza mediante clases presenciales, donde el docente transmite la información, siguiendo los esquemas tradicionales, siendo los recursos utilizados para el desenvolvimiento de la cátedra; la pizarra, marcador y presentaciones con diapositivas (Bayonès, 2010). Sin embargo, las nuevas técnicas de enseñanza consideran el uso de las TIC's para el proceso educativo (Adell, 1997), más aun cuando el país se encuentra en un proceso de transformación tecnológica, que ha hecho que los procesos contables y tributarios hayan sido automatizados, ejemplo de lo antes mencionado son la facturación electrónica, trámites gubernamentales en línea, entre otros.

La Tecnología tiene vital importancia en la educación superior (Cebián, 2003), porque hace posible que los estudiantes y docentes puedan simular a través de un programa contable, las transacciones diarias que tienen que registrar las empresas (Moreno \& Quiñonez, 2009).

Los estudiantes de la asignatura de contabilidad mantienen un alto índice de reprobación, el cual supera el $30 \%$. Esto podría deberse a desmotivación con la estrategia de enseñanza actual, o también a que la base de conocimientos que tienen los estudiantes no es la adecuada para el normal desenvolvimiento de la cátedra, lo cual es evidenciado por los docentes encargados de esta cátedra.

\subsection{Las tecnologías y el enfoque de aprendizaje interactivo}

Hace algunos años, según lo señala Marqués (2012) se introdujo un término, de cierta forma revolucionario, que se vería involucrado en entornos empresariales al igual que en entornos educativos. El término al cual se hace referencia es el de las tecnologías de información y comunicación, las cuales han tenido una acelerada evolución y posicionamiento en los diferentes ámbitos del mundo moderno.

Las tecnologías de la información y comunicación fueron rápidamente copando amplios espacios en empresas, instituciones y organismos a los largo de todo el mundo (Giner, 2004). Las instituciones educativas no fueron excluidas de estos avances a nivel tecnológico y poco a poco fueron adoptando también estas nuevas tecnologías tanto a nivel administrativo, como a nivel educativo, siendo en este último donde demostraron su alto grado de funcionabilidad y apli- cabilidad para la cuestión académica (Malagón, 2006).

El acceso a las tecnologías de información y comunicación por parte de los miembros de la comunidad educativa debe venir acompañado de un adecuado conocimiento sobre el manejo y el potencial de dichas tecnologías, dependiendo del ámbito específico donde se la quiera aplicar (Dussel \& Quevedo, 2011). Como parte de su explotación en los procesos de enseñanza aprendizaje, estas tecnologías representan la utilización de una cantidad de recursos importante, los cual impacta positivamente al proceso y a sus resultados, pero no se debe descuidar el impacto negativo asociado a la implementación de estas nuevas tecnologías en el proceso educativo (Severín, 2010).

En el ámbito educativo, las tecnologías de información y comunicación, ofrecen un sinnúmero de herramientas que pueden explotarse (Dorfsman, 2015); entre otras se pueden citar las plataformas virtuales, las aplicaciones para dispositivos electrónicos, el multimedia, las videoconferencias, los chats, las pizarras virtuales, las redes sociales, los libros electrónicos, los blogs, las wikis, la computación de nube (Sardelich, 2006).

Todos los recursos y herramientas dentro de las tecnologías de información y comunicación, pueden ser clasificados en recursos informativos, comunicacionales o de aprendizaje (Cacheiro M., 2011). Los recursos de información le permiten al usuario obtener datos sobre cualquier temática específica sin importar el lugar donde se encuentre y el momento en el que exista la necesidad (Medina, 2009). Los recursos de colaboración, le permiten al usuario la participación en diferentes trabajos y con diferentes personas sin la necesidad de estar físicamente presente, aportando sus ideas en tiempo real. Los recursos de aprendizaje son justamente los que posibilitan los procesos de aprendizaje como tal.

Ahora bien, el aprendizaje interactivo, que como concepto fue creado por Gumperz (1982), como una metodología de intervención para ayudar en procesos de adquisición de la comunicación y el lenguaje (Suárez \& Gros, 2013), está muy ligado con las TIC's, debido a que promueven una mejor interacción entre los alumnos y el profesor en ambientes de aprendizaje.

$\mathrm{El}$ aprendizaje interactivo requiere que tanto los alumnos como los profesores desarrollen nuevas competencias a nivel tecnológico y a nivel comunicativo (Mas Torelló, 2011), debido a que los trabajos se desarrollan de forma grupal 
y usualmente utilizan plataformas tecnológicas que permitan el trabajo grupal sin necesidad de que haya una interacción física de los miembros del grupo (Salinas, De Benito, \& Lizana, 2014).

Es importante anotar que tanto el uso de las TIC's, como la implementación del aprendizaje interactivo en el aula presentan como desafío el cambio de roles de docentes y estudiantes, los primeros pasan a ser facilitadores del proceso y los segundos se convierten en protagonistas de su propio aprendizaje (Benvenuto, 2003).

\section{METODOLOGÍA}

Para el presente artículo se utilizó un enfoque mixto de investigación. La parte cuantitativa, permitió recolectar información relacionada con la situación actual de los estudiantes que se encontraban cursando las asignaturas de Contabilidad 1 y Contabilidad 2, de la institución en estudio; la parte cualitativa, permitió conocer la opinión de los docentes de las asignaturas antes mencionadas.

En ambos casos los objetivos apuntaron a determinar la necesidad de tener acciones que impliquen la utilización de nuevas tecnologías, como es el caso del aprendizaje interactivo. Asimismo se hizo una búsqueda de datos secundarios internos sobre estadística de aprobados y reprobados en las asignaturas de Contabilidad 1 y 2, de igual manera se obtuvo datos sobre pruebas de diagnóstico realizadas al inicio del período.

La población para el análisis cuantitativo estuvo conformada por los estudiantes de las asignaturas de Contabilidad 1 y Contabilidad 2 de la carrera de Administración de Empresas, que totaliza 718 estudiantes.

Para el cálculo del tamaño de muestra se consideró una proporción poblacional ajustada del $50 \%$, un nivel de confianza del $95 \%$ y un error de no más del 5\%. A continuación se puede observar el cálculo realizado para la muestra de la parte cuantitativa:

$$
\begin{aligned}
& n=\frac{Z^{2} P Q N}{e^{2}(N-1)+Z^{2} P Q} \\
& n=\frac{(1,96)^{2} \times 0,5 \times 0,5 \times 718}{(0,05)^{2} \times 717+(1,96)^{2} \times 0,5 \times 0,5} \\
& n \approx 250
\end{aligned}
$$

El tamaño de muestra calculado fue de 250 estudiantes, a los cuales se les aplicó una encuesta, compuesta de siete preguntas cerradas, dispuestas de forma acorde a los objetivos planteados. Para determinar la cantidad de elementos muestrales en cada asignatura, se consideró un método de afijación proporcional. En la tabla I se puede observar la distribución de la muestra para los estudiantes de las asignaturas de Contabilidad 1 y 2 .

Tabla I. Distribución de la muestra.

\begin{tabular}{lccc}
\hline Asignatura & Población & Ponderación & Muestra \\
\hline Contabilidad 1 & 371 & $51,67 \%$ & 129 \\
Contabilidad 2 & 347 & $48,33 \%$ & 121 \\
\multicolumn{1}{c}{ Totales } & 718 & $100,00 \%$ & 250 \\
\hline
\end{tabular}

Fuente: Elaboración propia, a partir del Sistema Académico de la Universidad en estudio.

La población para el análisis cualitativo estuvo conformada por los docentes de las asignaturas de Contabilidad 1 y Contabilidad 2 de la carrera de Administración de Empresas, que totaliza 8 docentes. Para la muestra se utilizó un muestreo por conveniencia. El método de medición empleado fue una entrevista en profundidad.

\section{RESULTADOS}

\subsection{Encuestas a estudiantes de las asignaturas de Contabilidad 1 y 2}

La muestra considerada en el estudio estuvo caracterizada en términos de las variables sexo, asignatura y el número de veces que la ha to- 
mado. En cuanto a la variable sexo, la muestra estuvo conformada por un $70.4 \%$ de mujeres y un $29.6 \%$ de hombres. En cuanto a la asignatura, el $51.6 \%$ de la muestra estuvo constituida por estudiantes de Contabilidad 1 y el $48.4 \%$ por estudiantes de Contabilidad 2.

El 78\% de los encuestados, tal como se indica en el gráfico 1, se encontraban tomando la asignatura por primera vez, un $19.2 \%$ se encontraban repitiendo la asignatura por segunda vez y el otro $2.8 \%$ se encontraba repitiendo por tercera ocasión la asignatura. Cabe indicar que la asignatura de Contabilidad 1 presentó una mayor cantidad de estudiantes repetidores.

Gráfico 1: Número de veces que se ha tomado la asignatura.

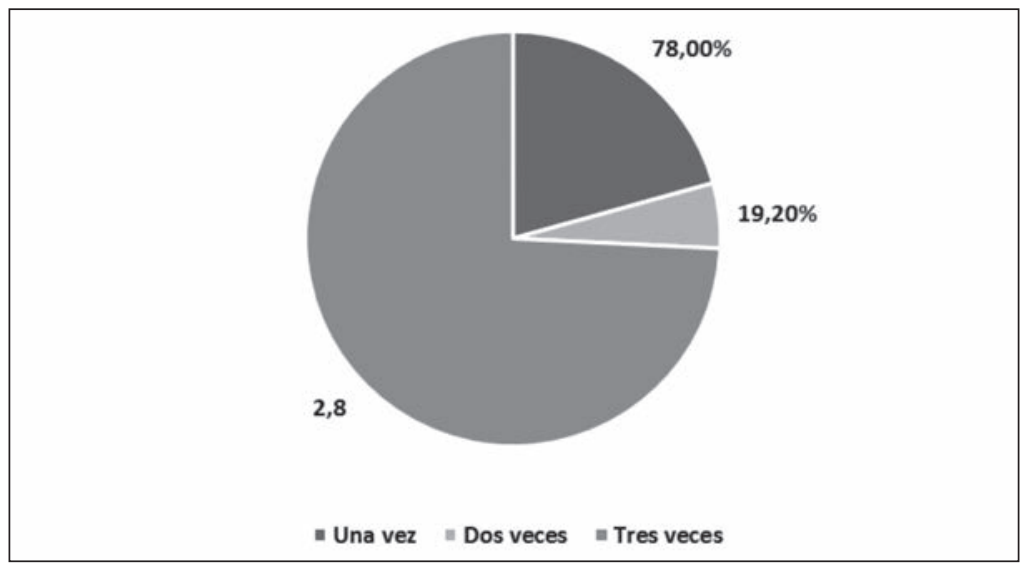

Fuente: Elaboración propia, a partir de los resultados de encuesta a estudiantes.

El $89.6 \%$ de los encuestados mostraron su agrado por la asignatura de contabilidad, mientras que el otro $10.4 \%$ mostró su desagrado por la asignatura. El 55.6\% de los encuestados indicó que el actual sistema motiva al aprendizaje de la materia, mientras que $44.4 \%$ consideró que los sistemas actuales no motivan al estudio de la asignatura.
El 74\% de los encuestados, tal como se muestra en el gráfico 2, considera que su nivel de manejo de las herramientas de Office es medio, $18.8 \%$ considera que su nivel de manejo es bajo y un $7.2 \%$ considera tener un nivel alto de manejo de estas herramientas.

Gráfico 2: Nivel de manejo de las herramientas de Office.

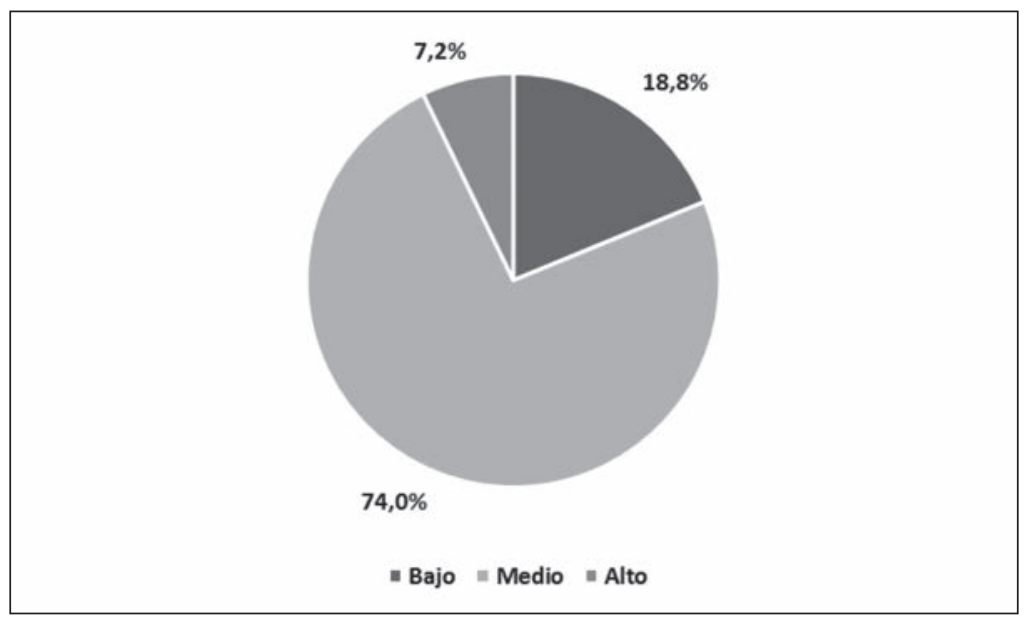

Fuente: Elaboración propia, a partir de los resultados de encuesta a estudiantes. 
El 92\% de los encuestados aseguró contar con un computador dentro de su domicilio, mientras que un $8 \%$ indicó no poseer computadora en su domicilio. El 87.2\% de los encuestados aseguró contar con acceso a Internet desde su domicilio, el resto indicó no tener acceso a la Internet.

El $94.42 \%$ de los encuestados considera que tener la posibilidad de practicar ejercicios contables a través de medios interactivos facilitaría el aprendizaje de la asignatura.

En la Tabla II se indican las actividades que mejorarían el aprendizaje, el $43.8 \%$ apuntó hacia tener horas en laboratorio, un $23.4 \%$ señaló la opción de tutorías virtuales, el $19.1 \%$ de las respuestas se encaminaron hacia la opción de tener ayudas interactivas en AVAC y el $13.6 \%$ subrayó la posibilidad de tener tareas con ayudas de programas descargables de la web.

Tabla II. Actividades encaminadas al mejoramiento del aprendizaje.

\begin{tabular}{|cl|r|r|r|}
\hline & \multicolumn{2}{|c|}{ Respuestas } & Porcentaje de \\
\cline { 3 - 4 } & \multicolumn{1}{|c|}{$\mathrm{N}$} & Porcentaje & \multicolumn{1}{c|}{ casos } \\
\hline Actividad $^{\text {a }}$ & $\begin{array}{l}\text { Horas de clase en } \\
\text { laboratorio }\end{array}$ & 183 & $43,8 \%$ & $73,2 \%$ \\
& $\begin{array}{l}\text { Deberes en programas } \\
\text { descargables }\end{array}$ & 57 & $13,6 \%$ & $22,8 \%$ \\
& $\begin{array}{l}\text { Ayudas interactivas en el } \\
\text { AVAC }\end{array}$ & 80 & $19,1 \%$ & $32,0 \%$ \\
Total & Tutoría pedagógica virtual & 98 & $23,4 \%$ & $39,2 \%$ \\
& & 418 & $100,0 \%$ & $167,2 \%$ \\
\hline
\end{tabular}

Fuente: Elaboración propia, a partir de los resultados de encuesta a estudiantes.

\subsection{Entrevistas}

Se planteó la realización de entrevistas a los docentes de las asignaturas de Contabilidad 1 y 2 de la carrera, la misma se basó en cinco preguntas abiertas de carácter exploratorio, buscando evaluar la motivación que tienen los estudiantes que toman las asignaturas de Contabilidad 1 y 2 y la percepción que tienen los docentes sobre el uso de herramientas tecnológicas y de aprendizaje interactivo y el impacto que podrían tener las mismas en la motivación de los estudiantes y las tasas de reprobación que experimenta la carrera en dichas asignaturas.

Los docentes opinaron que el principal motivo por los cuales los estudiantes no gustan de la contabilidad es por la falta de bases, lo cual hace que en muchos de los casos se sientan desmotivados y a la larga se pierdan al momento de profundizar en la materia. Los docentes concuerdan en que la motivación es importante no solo para asignaturas contables, sino para el resto de asignaturas del pensum académico; algunas de las estrategias que citan los docentes para motivar a sus estudiantes fueron, tener un trato amable con los estudiantes, acercar la teoría a la práctica contable con el propósito de que los estudiantes evidencien la importancia del aprendizaje de la contabilidad, se indica también que es importante el uso de herramientas tecnológicas que faciliten el aprendizaje y proporcione mayores recursos para que los estudiantes accedan y puedan practicar la asignatura.

Los docentes concuerdan en el hecho de que el uso de herramientas tecnológicas y de aprendizaje interactivo elevaría los niveles de motivación de los estudiantes, a la vez que ayudaría a disminuir las tasas de repitencia, debido a que complementarían las otras acciones que se han implementado, como son las tutorías, ayudantías y trabajo con la plataforma virtual.

Sobre las herramientas de aprendizaje interactivo propuestas por los docentes del área se encuentran las tutorías virtuales, los chats interactivos en horarios definidos con los docentes, la implementación de videos tutoriales elaborados por los docentes y el acceso a bibliografía electrónica.

Finalmente, los docentes concuerdan en que a pesar de que al inicio el uso de herramientas interactivas puede generar resistencia, en el mediano plazo se podrá evidenciar las ventajas 
dentro de la cátedra y esto despertará el interés de los docentes por usar dichas herramientas con lo cual habrá la necesidad de impartir capacitación focalizada en la implementación y uso de las herramientas de aprendizaje interactivo.

\subsection{Datos secundarios}

Debido al alto número de estudiantes que reprobaban las asignaturas de Contabilidad 1 y 2, en conversaciones realizadas con el cuerpo docente que imparte la materia, se decidió se tomen pruebas de diagnóstico a los estudiantes, con la finalidad de saber el grado de conocimiento con el que ellos ingresan al curso, lo cual a su vez le permite al docente tener una guía de cómo comenzar la práctica estudiantil a partir de estos conocimientos de base, los cuales en la mayoría de los grupos necesita ser reforzado.

El gráfico 3 muestra la cantidad de alumnos que rindieron la prueba de diagnóstico y los resultados obtenidos.

Gráfico 3: Calificaciones en pruebas diagnósticas para asignaturas de Contabilidad 1 y 2.

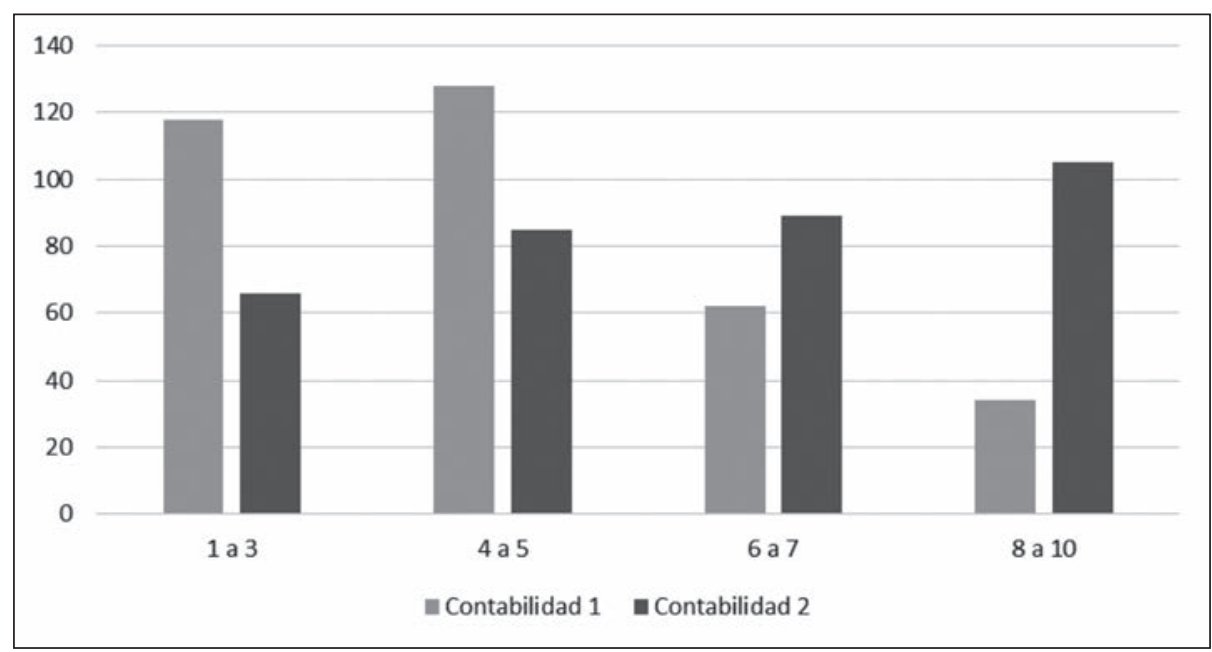

Fuente: Elaboración propia a partir de los resultados de encuesta a estudiantes.

En las pruebas diagnósticas realizadas por los estudiantes de Contabilidad 1, el 34.5\% obtuvo notas entre 1 y 3 , el $37.43 \%$ entre 4 y 5 y el otro $28.07 \%$ obtuvo notas por sobre el 5 . Por su parte de los estudiantes que realizaron la prueba de diagnóstico de Contabilidad 2, el 19.13\% tuvo notas entre 1 y 3 , el $24.64 \%$ notas entre 4 y 5 y el $56.23 \%$ restante notas por sobre los 5 puntos.

Se analizó la cantidad de alumnos inscritos en los tres últimos periodos, esto es, 44,45 y 46 para poder mostrar una tendencia del alto índice de estudiantes reprobados en las asignaturas de Contabilidad 1 y 2 de los estudiantes de la carrera de Administración de Empresas.

Tomando como argumento los datos que reposan en Dirección de Carrera de Administración de Empresas, para poder comprobar las tasas de reprobación de los estudiantes en la asignatura de contabilidad 1 y los diferentes docentes asignados a la cátedra, los mismos que se muestran gráficamente.

La Tabla III evidencia la cantidad de estudiantes que aprobaron, reprobaron o anularon de materia de los tres últimos periodos, de las asignaturas de contabilidad 1 y 2 .

En el período 44, se inscribieron 710 estudiantes en la asignatura de Contabilidad 1, de los cuales reprobaron la materia 287, lo cual representa el $40.42 \%$. En el periodo 45 tomaron la materia 360 estudiantes de los cuales 188 reprobaron la materia, siendo esto el $52.22 \%$ y en el periodo 46 hubo 753 inscritos, reprobando la materia 383 estudiantes, que representa el $50.86 \%$. 
Tabla III: Estadística de aprobación de la asignatura Contabilidad 1, períodos 44 al 46.

\begin{tabular}{|c|c|c|c|}
\hline Contabilidad I & & & \\
\hline Período & 44 & 45 & 46 \\
\hline Aprobados & 423 & 172 & 370 \\
\hline Reprobados & 287 & 188 & 383 \\
\hline Total & 710 & 360 & 753 \\
\hline \% Reprobados & $40,42 \%$ & $52,22 \%$ & $50,86 \%$ \\
\hline
\end{tabular}

Fuente: Elaboración propia a partir del Sistema Académico de la Universidad en estudio.

Para la asignatura de Contabilidad 2, tal como se muestra en la Tabla IV, se inscribieron 261 estudiantes durante el período 44, de los cuales reprobaron la materia 42 , lo cual representa el $16.09 \%$. En el periodo 45 tomaron la materia
443 estudiantes de los cuales 155 reprobaron la materia, siendo esto el $34.99 \%$ y en el periodo 46 hubo 248 inscritos, reprobando la materia 84 estudiantes, que representa el $33.87 \%$.

Tabla IV. Estadística de aprobación de la asignatura Contabilidad 2, períodos 44 al 46.

\begin{tabular}{|c|c|c|c|}
\hline Contabilidad II & & & \\
\hline Período & 44 & 45 & 46 \\
\hline Aprobados & 219 & 288 & 164 \\
\hline Reprobados & 42 & 155 & 84 \\
\hline Total & 261 & 443 & 248 \\
\hline$\%$ Reprobados & $16,09 \%$ & $34,99 \%$ & $33,87 \%$ \\
\hline
\end{tabular}

Fuente: Elaboración propia a partir del Sistema Académico de la Universidad en estudio.

Como se puede apreciar en el gráfico 4, existe un alto número de estudiantes que reprueban la materia, existiendo así la preocupación por parte de los docentes y autoridades de la carre- ra en que exista una mejora de los métodos de enseñanza actualmente impartidos, producto de esta investigación.

Gráfico 4: Evolución de las tasas de aprobación para asignaturas de Contabilidad 1 y 2.

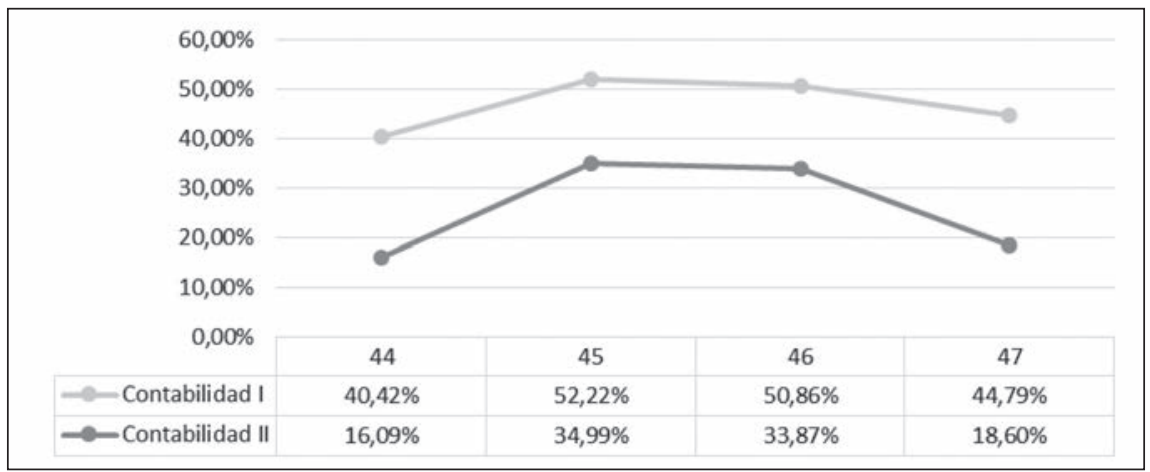

Fuente: Elaboración propia a partir del Sistema Académico de la Universidad en estudio. 


\section{DISCUSIÓN}

Los datos estadísticos de la institución muestran que las tasas de repitencia son altas para las asignaturas de Contabilidad 1 y 2, de hecho, la asignatura de Contabilidad 1 es la asignatura de primer semestre con mayor índice de reprobados, mientras que, en el segundo semestre, Contabilidad 2 se ubica en segundo lugar en los niveles de reprobación luego de Matemáticas 2.

Si bien es cierto en el período 46 se experimenta una disminución en las tasas de reprobación de las asignaturas contables, eso se debe a la implementación de nuevas estrategias de tutorías, pero fue en el período 47 que se experimentó una mejora más amplia debido a la utilización de laboratorios en clase, con computadores portátiles.

Las encuestas evidencias que existe un alto nivel de aceptación por parte de docentes y estudiantes para la aplicación de nuevas estrategias en la enseñanza de la contabilidad, que permitan a los docentes impartir la cátedra de forma más dinámica e interactiva.

A continuación, se hace referencia a algunas de las acciones que se pretenden implementar como parte de una nueva estrategia de enseñanza que incluye el uso de la tecnología y el aprendizaje interactivo.

La primera acción que se plantea es la continuidad de los laboratorios en aula, lo cual no solo demostró tener excelentes resultados elevando la motivación de los estudiantes, sino que permite que el conocimiento esté mejor afianzado en los estudiantes, debido a un trabajo en aula mucho más práctico. En el corto plazo la carrera planea la compra de un software que permita la realización de casos con datos contables y tributarios reales, lo cual permita el trabajo en grupos guiados por el docente.

Durante el período 47 también se integró un ayudante de cátedra para que pueda complementar las clases de los docentes del área, con lo cual la segunda acción a implementar es el uso de tutorías virtuales con la utilización de las opciones de chat presentes en el aula virtual de la institución.

Una tercera acción se desarrolla en conjunto con el equipo de coaching educativo, que se trata de un eje transversal de iniciativa estudiantil que pretende generar videos tutoriales los cuales se pueden encontrar en Youtube, así los docentes contarán con una herramienta de enseñanza adicional a la asignatura impartida en el aula. Para la implementación de esta acción el docente genera grupos de trabajo los mismos que se encargan de elaborar casos prácticos, en los cuales se utilicen los conocimientos impartidos en la asignatura y de esta forma los videos se encuentren disponibles para los estudiantes a través de la plataforma virtual.

La cuarta acción involucra la explotación de las herramientas tecnológicas presentes en la plataforma virtual:

- Foros abiertos: que sirvan para que el docente desarrolle temáticas inherentes a la asignatura y que generen dudas entre los estudiantes, así como para que los estudiantes expongan lo que han generado a través de los videos tutoriales.

- Evaluaciones automatizadas: que permitan afianzar los conocimientos en aquellas partes teóricas que son claves en la asignatura, como por ejemplo los planes de cuentas.

- Wikis: En las cuales los estudiantes podrán trabajar de forma colaborativa en el desarrollo de casos relacionados con la asignatura y dichos trabajos podrán estar disponibles a través de la plataforma virtual para los integrantes de otros grupos de trabajo.

Una última acción se enfoca en el establecimiento de una guía de talleres que se realizarían durante el transcurso del período académico, en los cuales los estudiantes conforman grupos en los cuales desarrollas casos prácticos de la materia aprendida en períodos cortos de tiempo. Los grupos se cambian cada cierto tiempo, para que las posibilidades de interacción entre todos los miembros del aula sean mejores.

\section{BIBLIOGRAFÍA}

Adell, J. (1997). Tendencias en educación en la sociedad de las tecnologìas de la informaciòn. Revista electrònica de tecnologìa educativa, 1-21.

Bayonès, M. (2010). La articulación en Contabilidad y las tecnologías de información y comunicación (tic). En búsqueda del aprendizaje significativo. Ciencias económicas, 47-61.

Benvenuto, A. (2003). Las tecnologías de información y comunicaciones (TIC) en la docencia universitaria. Theoría, 109-118.

Cacheiro, M. (2011). Recursos educativos TIC de 
información, colaboración y aprendizaje. Revista de Medios y Educación, 69-81.

Cebián, M. (2003). Enseñanza Virtual para la Innovación Universitaria. Madrid: S.A. de Ediciones.

Dorfsman, M. (2015). La profesión docente en contextos de cambio: el docente global en la sociedad de la información. Revista de Educación a Distancia - Docencia Universitaria en la Sociedad del Conocimiento, 1-23.

Dussel, I., \& Quevedo, L. (2011). Educación y nuevas tecnologías: los desafíos pedagógicos ante el mundo digital. Buenos Aires: Fundación Santillana.

Gallardo, E. (2012). Hablemos de estudiantes digitales y no de nativos digitales. UT. Revista de Ciències de l'Educació, 7-21.

Giner, F. (2004). Los sistemas de información en la sociedad del conocimiento. Madrid: ESIC Editorial.

Iglesias, M., \& Iglesias, P. (2016). La innovación evaluativa y el cambio de paradigma en la enseñanza contable. Revista Retos (12), 165-178.

Malagón, A. (2006). ¿Qué pueden aportar las tecnologías de la información y de la comunicación al campo educativo? Revista EAN, 185-200.

Marqués, P. (2012). Impacto de las TIC en la educación: funciones y limitaciones. Revista de investigación 3 Ciencias, 1-15.

Mas Torelló, O. (2011). El profesor universitario: Competencias y formación. Profesorado. Revista de currículum y formación del profesorado, 195-211.

Medina, A. (2009). Metodología didáctica para el desarrollo de planes de estudio en el EEES. En A. Medina, M. Sevillano, \& S. De la Torre, Una universidad para el s. XXI. Espacio Europeo de Educación Superior (EEES) (págs. 195-212). Madrid: Universitas.

Ministerio de Educación del Ecuador. (2016). Reglamento a la LOEI . Quito: Dirección Nacional de Normativa Jurídico Educativa.

Moreno, M., \& Quiñonez, D. (2009). La perspectiva didáctica de la estimulación motivacional en el proceso de enseñanza-aprendizaje. Revista Iberoamericana de Educación, 1-12.
Salinas, J., De Benito, B., \& Lizana, A. (2014). Competencias docentes para los nuevos escenarios de aprendizaje. Revista Interuniversitaria de Formación del Profesorado, 145-163.

Sardelich, M. (2006). Las nuevas tecnologías en educación: Aplicación e integración de las nuevas tecnologías en el desarrollo curricular. Madrid: Editorial Vigo.

Severín, E. (Febrero de 2010). Tecnologías de la información y la comunicación (TICs) en educación. Banco Interamericano de Desarrollo, División Educación, Notas técnicas \# 6, 2-39. Washington: BID.

Suárez, C., \& Gros, B. (2013). Aprender en red: de la interacción a la colaboración. Barcelona: Editorial UOC. 